\title{
Reliability optimization problems with multiple constraints under fuzziness
}

\author{
Neha Gupta $^{1} \cdot$ Sanam Haseen $^{1} \cdot$ Abdul Bari $^{1}$
}

Received: 1 November 2014/ Accepted: 7 June 2016/Published online: 18 June 2016

(c) The Author(s) 2016. This article is published with open access at Springerlink.com

\begin{abstract}
In reliability optimization problems diverse situation occurs due to which it is not always possible to get relevant precision in system reliability. The imprecision in data can often be represented by triangular fuzzy numbers. In this manuscript, we have considered different fuzzy environment for reliability optimization problem of redundancy. We formulate a redundancy allocation problem for a hypothetical series-parallel system in which the parameters of the system are fuzzy. Two different cases are then formulated as non-linear programming problem and the fuzzy nature is defuzzified into crisp problems using three different defuzzification methods viz. ranking function, graded mean integration value and $\alpha$-cut. The result of the methods is compared at the end of the manuscript using a numerical example.
\end{abstract}

Keywords Reliability optimization problem · Redundancy allocation problem - Triangular fuzzy numbers · Ranking function $\cdot$ Graded mean integration value $\cdot \alpha$-Cut

\section{Introduction}

Reliability theory has evolved through the demands of modern technology, particularly due to the experiences gained from complex military systems in World War II.

Neha Gupta

ngngupta4@gmail.com

Sanam Haseen

sanam.haseen@gmail.com

Abdul Bari

bariamu2k3@yahoo.co.in

1 Department of Statistics and O.R., Aligarh Muslim University, Aligarh 202002, UP, India
Most of the problems attempted earlier were machine maintenance and system reliability in military systems. Today reliability is considered in almost all engineering system designs. Problem of the reliability in various designs of system have been explored by Tillman et al. (1980), Misra (1986), Kuo et al. (2001), Taghizadeh and Hafezi (2012), Yusuf (2014), Rao and Naikan (2014), Bourezg and Meglouli (2015), and many more.

One such classical problem is the reliability optimization problem. In reliability optimization problem, often the objective is to maximize the system reliability or system availability or minimize the cost of system subject to constraints including budget restrictions, reliability requirements, and other considerations such as volume and weight. The design parameters or decision variables may be component reliability value, number of redundancies, or arrangement of known components. Some of the authors who work on reliability optimization problems with multiple constraints incorporated are Sasaki and Shingai (1983), Chern and Jan (1986), Sung and Cho (2000) etc.

This manuscript considers a system for redundancy allocation in reliability optimization problem. Redundancy allocation problem (RAP) optimizes reliability in seriesparallel systems. Researchers have been trying to maximize the reliability of RAP for complex reliability designs. Khalili-Damghani and Amiri (2012) used a hybrid efcient epsilon-constraint, multi-start partial bound enumeration algorithm to solve a binary-state multi-objective reliability redundancy allocation series parallel problem. A problem of reliability redundancy allocation has been solved using particle swarm optimization method by Khalili-Damghani et al. (2013). Similarly, a multi-objective redundancy allocation problem has been solved by Khalili-Damghani et al. (2014) using a decision support system. Real-life problems such as projects and case studies have also been 
studied for maximizing reliability in various systems. Dolatshahi-Zand and Khalili-Damghani (2015) designed the supervisory control and data acquisition (SCADA) water resource management control center using a bi-objective redundancy allocation problem and particle swarm optimization. A case study of the investigation of supply chain's reliability measure has been done by Taghizadeh and Hafezi (2012). Bourezg and Meglouli (2015) has done a reliability assessment of power distribution systems.

In conventional optimization problems decision maker (DM) assumes that the parameters or goals of the problem are precisely known in advance. But in real world situations this assumption is not always true, there are incompleteness and unreliability of input information. In such situations, the need to use fuzzy concept occurs. In reliability optimization problems diverse situations occur due to which it is not always possible to get relevant precise data for the reliability system. In such situations, fuzzy numbers can represent the data for the reliability system. Some author have used fuzzy sets theory in reliability optimization problems such as Huang (1996) solve the problem of multi-objective optimization problem of series system reliability using fuzzy sets theory, Mahapatra and Roy (2006) used generalized triangular fuzzy numbers to maximize the reliability of series and complex systems under the cost constraint, Mahapatra and Roy (2009) find the fuzzy reliability of a series and a parallel system using triangular intuitionistic fuzzy numbers, Mahapatra and Roy (2011) used fuzzy parametric geometric programming to analyze the reliability and cost of series system models. Besides them Bortolan and Degani (1982), Park (1987), Liou (1992), Hsieh and Chen (1999) are few more authors who worked in this field.

In this manuscript, we have formulated reliability optimization problem with multiple constraints. In real-life system reliability problems data is not always precise due to some uncontrollable and unavoidable situations. Therefore, to deal with imprecise data we choose fuzzy theory concept and formulate crisp, fuzzy, and fully fuzzy nonlinear integer programming problems. Fuzzy problems are defuzzified by ranking function, graded mean integration value and $\alpha$-cut methods. To demonstrate the proposed problems a numerical problem has been solved by an optimization software LINGO (2013).

\section{Prerequisite mathematics}

Some preliminary definitions are reviewed here from Mahapatra and Roy (2006), Bector and Chandra (2005).

Fuzzy theory was initiated by Zadeh (1965) with his seminal paper "Fuzzy Sets", in which he represents impreciseness or vagueness in everyday life as a mathematical model.

Fuzzy set: A fuzzy set $\tilde{A}$ in a universe of discourse $\mathrm{X}$ is defined as the following set of pairs $\tilde{A}=\left\{\left(x, \mu_{\tilde{A}}(x)\right): x \in X\right\}$. Here $\mu_{\tilde{A}}: X \rightarrow[0,1]$ is a mapping called the membership function of the fuzzy set $\tilde{A}$ and $\mu_{\tilde{A}}$ is called the membership value or degree of membership of $x \in X$ in the fuzzy set $\tilde{A}$. The larger $\mu_{\tilde{A}}$ is the stronger the grade of membership in $\tilde{A}$.

Fuzzy number: A fuzzy set $\mathrm{A}$ in $\mathbb{R}$ is called a fuzzy number if it satisfies the following conditions

- A is normal,

- $A_{\alpha}$ is a closed interval for every $\alpha \in(0,1]$,

- the support of A is bounded.

$\alpha$-cut The $\alpha$-cut of a fuzzy set A of $\mathrm{X}$ is a nonfuzzy set denoted by ${ }^{\alpha} A$ defined by a subset of all elements $x \in X$, such that their membership functions exceed or equal to a real number $\alpha \in[0,1]$, that is

$$
{ }^{\alpha} A=\left[x: \mu_{\tilde{A}}(x) \geq \alpha, \alpha \in[0,1], \forall x \in X\right]
$$

\section{Basic definitions and arithmetic operations}

In this section, we have reviewed some basic definitions and arithmetic operations of fuzzy numbers Bansal (2011), Aggarwal and Sharma (2013).

Definition 1 A fuzzy number $\tilde{A}=\left(a, a^{\prime}, a^{\prime \prime}\right)$ is said to be a triangular fuzzy number (TFN) if its membership function is given by

$\mu_{\tilde{A}}(x)=\left\{\begin{array}{cl}\frac{x-a}{a^{\prime}-a} & \text { if } a \leq x \leq a^{\prime} \\ \frac{x-a^{\prime \prime}}{a^{\prime}-a^{\prime \prime}} & \text { if } a^{\prime} \leq x \leq a^{\prime \prime} \\ 0 & \text { otherwise }\end{array}\right.$

Remark TFN denotes the set of all triangular fuzzy numbers.

Definition 2 A triangular fuzzy number $\tilde{A}=\left(a, a^{\prime}, a^{\prime \prime}\right)$ is said to be non-negative fuzzy number if and only if $a \geq 0$.

Definition 3 Two triangular fuzzy numbers $\tilde{A}=$ $\left(a, a^{\prime}, a^{\prime \prime}\right)$ and $\tilde{B}=\left(b, b^{\prime}, b^{\prime \prime}\right)$ are said to be equal if and only if $a=b, a^{\prime}=b^{\prime}, a^{\prime \prime}=b^{\prime \prime}$.

Arithmetic operations of triangular fuzzy numbers

Let $\tilde{A}=\left(a, a^{\prime}, a^{\prime \prime}\right)$ and $\tilde{B}=\left(b, b^{\prime}, b^{\prime \prime}\right)$ be two triangular fuzzy numbers then

1. Addition: $\tilde{A} \oplus \tilde{B}=\left(a, a^{\prime}, a^{\prime \prime}\right) \oplus\left(b, b^{\prime}, b^{\prime \prime}\right) \sim=(a+b$, $\left.a^{\prime}+b^{\prime}, a^{\prime \prime}+b^{\prime \prime}\right)$ 
2. Multiplication: Let $\tilde{B}=\left(b, b^{\prime}, b^{\prime \prime}\right)$ be a non-negative triangular fuzzy number then

$$
\tilde{A} \otimes \tilde{B} \cong\left\{\begin{array}{cc}
\left(a b, a^{\prime} b^{\prime}, a^{\prime \prime} b^{\prime \prime}\right) & \text { if } a \geq 0 \\
\left(a b^{\prime \prime}, a^{\prime} b^{\prime}, a^{\prime \prime} b^{\prime \prime}\right) & \text { if } a<0, a^{\prime \prime} \geq 0 \\
\left(a b^{\prime \prime}, a^{\prime} b^{\prime}, a^{\prime \prime} b\right) & a^{\prime \prime}<0
\end{array}\right.
$$

3. Exponential: The exponential of an arbitrary triangular fuzzy number can be obtained directly as:

$$
e^{\left(a, a^{\prime}, a^{\prime \prime}\right)}=\left(e^{a}, e^{a^{\prime}}, e^{a^{\prime \prime}}\right)
$$

4. Power function: The power function of an arbitrary triangular fuzzy number can be obtained directly as:

$$
x^{\left(a, a^{\prime}, a^{\prime \prime}\right)}=\left(x^{a}, x^{a^{\prime}}, x^{a^{\prime \prime}}\right)
$$

\section{Mathematical model}

Consider a system which requires to perform a sequence of identical missions after every given (fixed) period. The system consists of several subsystems. Let us consider a system reliability of $n$-stage series system with redundant units in parallel. It is also assumed that in each stage, different types of components can be used as design alternatives. Now, the nonlinear integer programming problem can be formulated as:

$$
\left.\begin{array}{ll}
\text { Maximize } R_{\mathrm{S}} & =\prod_{i=1}^{n}\left[1-\prod_{j=1}^{l_{i}}\left(1-R_{i j}\right)^{x_{i j}}\right] \\
\text { subject to } & \sum_{i=1}^{n} \sum_{j=1}^{l_{i}} c_{i j} x_{i j} \leq C_{0} \\
& \sum_{i=1}^{n} \sum_{j=1}^{l_{i}} w_{i j} x_{i j} \leq W_{0} \\
& \sum_{j=1}^{l_{i}} x_{i j} \geq 1 ; i=1,2, \ldots, n \\
& x_{i j} \geq 0, x_{i j} \text { integers, } 1 \leq i \leq n, 1 \leq j \leq l_{i}
\end{array}\right\}
$$

where $n$ is the number of subsystems, $x_{i j}$ is the number of the redundant components of design alternative $j$ in stage $i$, $c_{i j}$ is the cost of design alternative $j$ in stage $i, w_{i j}$ weight of design alternative $j$ in stage $i, l_{i}$ is the number of design alternatives in subsystem $i, R_{i j}$ is the reliability of design alternative $j$ in stage $i, R_{\mathrm{s}}$ is the reliability of the system, $C_{0}$ is the cost limitations, $W_{0}$ is the weight limitations.

\section{Fuzzy model}

Generally, a DM assumes before solving a reliability optimization problem that the coefficients of components are precisely known in advance. But in real world, several diverse situation occurs such as uncertain judgements, unpredictable conditions or human error, incomplete knowledge and information etc, due to which it is not necessary that we get a relevant precise data for the reliability system. Such type of impreciseness of any data can be represented in different ways. Among these, one way is to represent the imprecise data by fuzzy number. Therefore, the problem (1) can be reformulated as a fuzzy nonlinear integer programming problem as follows:

Type I: In this model, the cost and weight of design alternative $j$ in stage $i$, system cost and system weight limitations are considered as imprecise and assume them as TFNs.

$$
\left.\begin{array}{ll}
\text { Maximize } R_{\mathrm{s}} & =\prod_{i=1}^{n}\left[1-\prod_{j=1}^{l_{i}}\left(1-R_{i j}\right)^{x_{i j}}\right] \\
\text { subject to } \quad & \sum_{i=1}^{n} \sum_{j=1}^{l_{i}}\left(c_{i j}, c_{i j}^{\prime}, c_{i j}^{\prime \prime}\right) \otimes x_{i j} \leq\left(C_{0}, C_{0}^{\prime}, C_{0}^{\prime \prime}\right) \\
& \sum_{i=1}^{n} \sum_{j=1}^{l_{i}}\left(w_{i j}, w_{i j}^{\prime}, w_{i j}^{\prime \prime}\right) \otimes x_{i j} \leq\left(W_{0}, W_{0}^{\prime}, W_{0}^{\prime \prime}\right) \\
& \sum_{j=1}^{l_{i}} x_{i j} \geq 1 ; i=1,2, \ldots, n \\
& x_{i j} \geq 0, \\
& x_{i j} \text { integers, } 1 \leq i \leq n, 1 \leq j \leq l_{i}
\end{array}\right\} .
$$

Type II: In this model, we consider the configuration stage of reliability system. In real world situations, at this stage it is not necessary that a precise or certain data is known in advance. Therefore, in that case we assume a fully fuzzy reliability optimization problem which is formulated as follows:

$$
\left.\begin{array}{ll}
\text { Maximize } \tilde{R}_{\mathrm{S}} & =\prod_{i=1}^{n}\left[1-\prod_{j=1}^{l_{i}}\left(1-\left(R_{i j}, R_{i j}^{\prime}, R_{i j}^{\prime \prime}\right)\right)^{\left(x_{i j}, x_{i j}^{\prime}, x_{i j}^{\prime \prime}\right)}\right] \\
\text { subject to } \quad & \sum_{i=1}^{n} \sum_{j=1}^{l_{i}}\left(c_{i j}, c_{i j}^{\prime}, c_{i j}^{\prime \prime}\right) \otimes\left(x_{i j}, x_{i j}^{\prime}, x_{i j}^{\prime \prime}\right) \leq\left(C_{0}, C_{0}^{\prime}, C_{0}^{\prime \prime}\right) \\
& \sum_{i=1}^{n} \sum_{j=1}^{l_{i}}\left(w_{i j}, w_{i j}^{\prime}, w_{i j}^{\prime \prime}\right) \otimes\left(x_{i j}, x_{i j}^{\prime}, x_{i j}^{\prime \prime}\right) \leq\left(W_{0}, W_{0}^{\prime}, W_{0}^{\prime \prime}\right) \\
& \sum_{j=1}^{l_{i}}\left(x_{i j}, x_{i j}^{\prime}, x_{i j}^{\prime \prime}\right) \geq 1 ; i=1,2, \ldots, n \\
& \left(x_{i j}, x_{i j}^{\prime}, x_{i j}^{\prime \prime}\right) \geq 0
\end{array}\right\} .
$$




\section{Different defuzzification procedures}

In this section, we defuzzify the triangular fuzzy numbers. Actually defuzzification is the conversion of fuzzy number to precise or crisp number. There are several methods to do such type of conversion. Here we use three types of defuzzification, first one is followed by Kumar and Kaur (2011), second by Sen and Bhunia (2014) and third by Pramanik and Roy (2008).

\section{Ranking function procedure}

In this section fuzzy non linear integer programming problem has been defuzzified into crisp nonlinear integer programming problem using ranking function. A ranking function is a function $\mathfrak{R}: F(R) \rightarrow R$, where $F(R)$ is the set of fuzzy numbers defined on the set of real numbers, which maps each fuzzy number into the real line, where a natural order exists. Let $\tilde{A}=\left(a, a^{\prime}, a^{\prime \prime}\right)$ be a triangular fuzzy number then $\mathfrak{R}(\tilde{A})=\frac{a+2 a^{\prime}+a^{\prime \prime}}{4}$.

Now using the ranking function of triangular fuzzy number we get the crisp problem as follows:

Type I

$$
\left.\begin{array}{ll}
\text { Maximize } R_{\mathrm{s}} & =\prod_{i=1}^{n}\left[1-\prod_{j=1}^{l_{i}}\left(1-R_{i j}\right)^{x_{i j}}\right] \\
\text { subject to } & \sum_{i=1}^{n} \sum_{j=1}^{l_{i}} \mathfrak{R}\left(c_{i j}, c_{i j}^{\prime}, c_{i j}^{\prime \prime}\right) x_{i j} \leq \mathfrak{R}\left(C_{0}, C_{0}^{\prime}, C_{0}^{\prime \prime}\right) \\
& \sum_{i=1}^{n} \sum_{j=1}^{l_{i}} \mathfrak{R}\left(w_{i j}, w_{i j}^{\prime}, w_{i j}^{\prime \prime}\right) x_{i j} \leq \mathfrak{R}\left(W_{0}, W_{0}^{\prime}, W_{0}^{\prime \prime}\right) \\
& \sum_{j=1}^{l_{i}} x_{i j} \geq 1 ; i=1,2, \ldots, n \\
& x_{i j} \geq 0
\end{array}\right\}
$$

Similarly, fully fuzzy problem in Type II also converted into crisp or deterministic form as follows:

Type II

$$
\left.\begin{array}{ll}
\text { Maximize } \mathfrak{R}\left(\tilde{R}_{\mathrm{s}}\right) & =\prod_{i=1}^{n}\left[1-\prod_{j=1}^{l_{i}}\left(1-\mathfrak{R}\left(R_{i j}, R_{i j}^{\prime}, R_{i j}^{\prime \prime}\right)\right)^{\Re\left(x_{i j}, x_{i j}^{\prime}, x_{i j}^{\prime \prime}\right)}\right] \\
\text { subject to } & \sum_{i=1}^{n} \sum_{j=1}^{l_{i}} \mathfrak{R}\left(c_{i j}, c_{i j}^{\prime}, c_{i j}^{\prime \prime}\right) \mathfrak{R}\left(x_{i j}, x_{i j}^{\prime}, x_{i j}^{\prime \prime}\right) \leq \mathfrak{R}\left(C_{0}, C_{0}^{\prime}, C_{0}^{\prime \prime}\right) \\
& \sum_{i=1}^{n} \sum_{j=1}^{l_{i}} \mathfrak{R}\left(w_{i j}, w_{i j}^{\prime}, w_{i j}^{\prime \prime}\right) \mathfrak{R}\left(x_{i j}, x_{i j}^{\prime}, x_{i j}^{\prime \prime}\right) \leq \mathfrak{R}\left(W_{0}, W_{0}^{\prime}, W_{0}^{\prime \prime}\right) \\
& \sum_{j=1}^{l_{i}} \mathfrak{R}\left(x_{i j}, x_{i j}^{\prime}, x_{i j}^{\prime \prime}\right) \geq 1 ; i=1,2, \ldots, n \\
& \Re\left(x_{i j}, x_{i j}^{\prime}, x_{i j}^{\prime \prime}\right) \geq 0
\end{array}\right\}
$$

\section{Graded mean integration value (GMIV) procedure}

According to the Chen and Hsieh (1999) and Sen and Bhunia (2014) if $\tilde{A}$ is triangular fuzzy number with membership function $\mu_{\tilde{A}}(x)$, the graded mean integral value $P(\tilde{A})$ of $\tilde{A}$ is given by

$$
\begin{aligned}
P(\tilde{A}) & =\frac{\int_{0}^{1} x\left\{(1-w) L^{-1}(x)+w R^{-1}(x)\right\} \mathrm{d} x}{\int_{0}^{1} x \mathrm{~d} x} \\
& =\frac{\int_{0}^{1} x\left[\frac{a+a^{\prime \prime}+\left(2 a^{\prime}-a-a^{\prime \prime}\right) x}{2}\right] \mathrm{d} x}{\int_{0}^{1} x \mathrm{~d} x} \\
P(\tilde{A}) & =\frac{a+4 a^{\prime}+a^{\prime \prime}}{6}
\end{aligned}
$$

where $w \in[0,1]$ is the pre-assigned parameter refers the degree of optimism.

Using GMIV procedure, the problems represented by Eqs. (2) and (3) can be transformed into following problems:

Type I

$$
\left.\begin{array}{ll}
\text { Maximize } R_{\mathrm{S}} & =\prod_{i=1}^{n}\left[1-\prod_{j=1}^{l_{i}}\left(1-R_{i j}\right)^{x_{i j}}\right] \\
\text { subject to } & \sum_{i=1}^{n} \sum_{j=1}^{l_{i}}\left(\frac{c_{i j}+4 c_{i j}^{\prime}+c_{i j}^{\prime \prime}}{6}\right) x_{i j} \leq\left(\frac{C_{0}+4 C_{0}^{\prime}+C_{0}^{\prime \prime}}{6}\right) \\
& \sum_{i=1}^{n} \sum_{j=1}^{l_{i}}\left(\frac{w_{i j}+4 w_{i j}^{\prime}+w_{i j}^{\prime \prime}}{6}\right) x_{i j} \leq\left(\frac{W_{0}+4 W_{0}^{\prime}+W_{0}^{\prime \prime}}{6}\right) \\
& \sum_{j=1}^{l_{i}} x_{i j} \geq 1 ; i=1,2, \ldots, n \\
& x_{i j} \geq 0, x_{i j} \text { integers, } 1 \leq i \leq n, 1 \leq j \leq l_{i}
\end{array}\right\} .
$$

Type II

$$
\left.\begin{array}{rl}
\text { Maximize } R_{\mathrm{s}}= & \prod_{i=1}^{n}\left[1-\prod_{j=1}^{l_{i}}\left(1-\left(\frac{R_{i j}+4 R_{i j}^{\prime}+R_{i j}^{\prime \prime}}{6}\right) \frac{x_{i j}+4 x_{i j}^{\prime}+x_{i j}^{\prime \prime}}{6}\right)\right] \\
\text { subject to } \quad & \sum_{i=1}^{n} \sum_{j=1}^{l_{i}}\left(\frac{c_{i j}+4 c_{i j}^{\prime}+c_{i j}^{\prime \prime}}{6}\right)\left(\frac{x_{i j}+4 x_{i j}^{\prime}+x_{i j}^{\prime \prime}}{6}\right) \leq\left(\frac{C_{0}+4 C_{0}^{\prime}+C_{0}^{\prime \prime}}{6}\right) \\
& \sum_{i=1}^{n} \sum_{j=1}^{l_{i}}\left(\frac{w_{i j}+4 w_{i j}^{\prime}+w_{i j}^{\prime \prime}}{6}\right)\left(\frac{x_{i j}+4 x_{i j}^{\prime}+x_{i j}^{\prime \prime}}{6}\right) \leq\left(\frac{W_{0}+4 W_{0}^{\prime}+W_{0}^{\prime \prime}}{6}\right) \\
& \sum_{j=1}^{l_{i}}\left(\frac{x_{i j}+4 x_{i j}^{\prime}+x_{i j}^{\prime \prime}}{6}\right) \geq 1 ; i=1,2, \ldots, n \\
& \left(\frac{x_{i j}+4 x_{i j}^{\prime}+x_{i j}^{\prime \prime}}{6}\right) \geq 0, \text { and integers } 1 \leq i \leq n, 1 \leq j \leq l_{i}
\end{array}\right\}
$$

\section{$\alpha$-Cut procedure}

Let us suppose that the problem (2) and (3) has fuzzy coefficients having possibilistic distributions. Assume that $\mathrm{X}$ is the solution of the problems, where $\alpha \in[0,1]$ 
represents the level of possibility at which all fuzzy coefficients are feasible.

An $\alpha$-cut of a fuzzy number $\tilde{A}$ can be represented by the following interval,

$$
\begin{aligned}
{ }^{\alpha}(\tilde{A}) & =\left[{ }^{\alpha}(\tilde{A})^{L}, \alpha(\tilde{A})^{U}\right] \\
& =\left[\left(a^{\prime}-a\right) \alpha+a,-\left(a^{\prime \prime}-a^{\prime}\right) \alpha+a^{\prime \prime}\right]
\end{aligned}
$$

Now as the coefficients $\tilde{R}_{i j}$ of the objective function are fuzzy numbers, $\alpha$-cut of $\tilde{R}_{i j}$ can be defined as:

${ }^{\alpha}\left(\tilde{R}_{i j}\right)=\left\{S\left(\tilde{R}_{i j}\right) \mid \mu_{\tilde{R}_{i j}} \geq \alpha, \alpha \in[0,1]\right\}$

where $S\left(\tilde{R}_{i j}\right)$ is the support of $\tilde{R}_{i j}$ and ${ }^{\alpha}\left(\tilde{R}_{i j}\right)$ can be represented by the closed interval $\left[{ }^{\alpha}\left(\tilde{R}_{i j}\right)^{L},{ }^{\alpha}\left(\tilde{R}_{i j}\right)^{U}\right]$.

Then the lower and upper bound of the respective $\alpha$-cuts of the objective function are defined as:

$$
\begin{aligned}
& \left.{ }^{\alpha}\left(\tilde{R}_{i j}\right)^{L}=\prod_{i=1}^{n}\left[1-\prod_{j=1}^{l_{i}}\left(1-\left({ }^{\alpha}\left(\tilde{R}_{i j}\right)^{L}\right)^{\alpha}{ }^{\alpha} \tilde{\tilde{x}}_{i j}\right)^{L}\right)\right] \\
& { }^{\alpha}\left(\tilde{R}_{i j}\right)^{U}=\prod_{i=1}^{n}\left[1-\prod_{j=1}^{l_{i}}\left(1-\left({ }^{\alpha}\left(\tilde{R}_{i j}\right)^{U}\right)^{\alpha}{ }^{\alpha}\left(\tilde{x}_{i j}\right)^{U}\right)\right]
\end{aligned}
$$

Next, for a prescribed value of $\alpha$, to construct a membership function for maximization type objective function, $\tilde{R}_{\mathrm{s}}$, can be replaced by the upper bound of its $\alpha$-cut, that is

$$
{ }^{\alpha}\left(\tilde{R}_{i j}\right)^{U}=\prod_{i=1}^{n}\left[1-\prod_{j=1}^{l_{i}}\left(1-\left({ }^{\alpha}\left(\tilde{R}_{i j}\right)^{U}\right)^{\alpha}\left(\tilde{x}_{i j}\right)^{U}\right)\right]
$$

For inequality constraints

$$
\begin{aligned}
& \sum_{j=1}^{q} \tilde{A}_{i j} X_{j} \geq \tilde{B}_{i}, i=1,2, \ldots, p_{1} \\
& \sum_{j=1}^{q} \tilde{A}_{i j} X_{j} \leq \tilde{B}_{i}, i=p_{1}+1, \ldots, p_{2}
\end{aligned}
$$

can be rewritten by the following constraints:

$$
\begin{aligned}
& \sum_{j=1}^{q}{ }^{\alpha}\left(\tilde{A}_{i j}\right)^{U} X_{j} \geq^{\alpha}\left(\tilde{B}_{i}\right)^{L}, i=1,2, \ldots, p_{1} \\
& \sum_{j=1}^{q}{ }^{\alpha}\left(\tilde{A}_{i j}\right)^{L} X_{j} \leq^{\alpha}\left(\tilde{B}_{i}\right)^{U}, i=1,2, \ldots, p_{1}
\end{aligned}
$$

Therefore, for a prescribed value of $\alpha$, the problems represented by Eqs. (2), (3) can be transformed to the following problems:

Type I

$$
\left.\begin{array}{ll}
\text { Maximize } R_{\mathrm{S}} & =\prod_{i=1}^{n}\left[1-\prod_{j=1}^{l_{i}}\left(1-R_{i j}\right)^{x_{i j}}\right] \\
\text { subject to } & \sum_{i=1}^{n} \sum_{j=1}^{l_{i}} \alpha\left(\tilde{c}_{i j}\right)^{L} x_{i j} \leq \alpha\left(\widetilde{C}_{0}\right)^{U} \\
& \sum_{i=1}^{n} \sum_{j=1}^{l_{i}} \alpha\left(\tilde{w}_{i j}\right)^{L} x_{i j} \leq \alpha\left(\widetilde{W}_{0}\right)^{U} \\
& \sum_{j=1}^{l_{i}} x_{i j} \geq 1 ; i=1,2, \ldots, n \\
& x_{i j} \geq 0, x_{i j} \text { integers, } 1 \leq i \leq n, 1 \leq j \leq l_{i}
\end{array}\right\}
$$

Type II

$$
\left.\begin{array}{ll}
\text { Maximize } R_{\mathrm{s}} & =\prod_{i=1}^{n}\left[1-\prod_{j=1}^{l_{i}}\left(1-\left({ }^{\alpha}\left(\tilde{R}_{i j}\right)^{U}\right)^{\alpha}\left(\tilde{x}_{i j}\right)^{U}\right)\right] \\
\text { subject to } \quad & \sum_{i=1}^{n} \sum_{j=1}^{l_{i}} \alpha\left(\tilde{c}_{i j}\right)^{L \alpha}\left(\tilde{x}_{i j}\right)^{L} \leq{ }^{\alpha}\left(\widetilde{C}_{0}\right) \\
& \sum_{i=1}^{n} \sum_{j=1}^{l_{i}} \alpha\left(\tilde{w}_{i j}\right)^{L \alpha}\left(\tilde{x}_{i j}\right)^{L} \leq^{\alpha}\left(\widetilde{W}_{0}\right)^{U} \\
& \sum_{j=1}^{l_{i}} \alpha\left(\tilde{x}_{i j}\right)^{U} \geq 1 ; i=1,2, \ldots, n \\
& \alpha\left(\tilde{x}_{i j}\right)^{U} \geq 0, \text { and integers, } 1 \leq i \leq n, 1 \leq j \leq l_{i}
\end{array}\right\}
$$

\section{Illustrative example}

To illustrate the proposed problem and solution procedure we consider a numerical example from Chern and Jan (1986). Consider a 3-stage series system with redundant units in parallel (1-out-of-3: G stage) and assume that in each stage, different types of components can be used as design alternatives. Here the system budget and system weight for the crisp data is $C_{0}=$ 30 and $W_{0}=17$ and for the fuzzy data is $\tilde{C}_{0}=(26,30,33)$ and $\tilde{W}_{0}=(14,17,19)$. Rest of the data required to formulate the problem are given in the tables below:

Using the data in 1, NLIPP will be: 


$$
\left.\begin{array}{c}
\text { Maximize } R_{\mathrm{S}}=\left(1-(1-0.99)^{x_{11}}(1-0.95)^{x_{12}}(1-0.92)^{x_{13}}\right) \\
\left(1-(1-0.98)^{x_{21}}(1-0.8)^{x_{22}}(1-0.90)^{x_{23}}\right)\left(1-(1-0.98)^{x_{31}}(1-0.92)^{x_{32}}\right) \\
\text { subject to } 4 x_{11}+13 x_{12}+7 x_{13}+8 x_{21}+3 x_{22}+3 x_{23}+11 x_{31}+5 x_{32} \leq 30 \\
2 x_{11}+3 x_{12}+5 x_{13}+3 x_{21}+3 x_{22}+9 x_{23}+4 x_{31}+6 x_{32} \leq 17 \\
x_{11}+x_{12}+x_{13}+x_{21}+x_{22}+x_{23}+x_{31}+x_{32} \geq 1 \\
x_{11}, x_{12}, x_{13}, x_{21}, x_{22}, x_{23}, x_{31}, x_{32} \geq 0, \text { integers }
\end{array}\right\}
$$

Using the data in 2, FNLIPP will be:

$$
\begin{gathered}
\text { Maximize } R_{\mathrm{s}}=\left(1-(1-0.99)^{x_{11}}(1-0.95)^{x_{12}}(1-0.92)^{x_{13}}\right) \\
\left(1-(1-0.98)^{x_{21}}(1-0.8)^{x_{22}}(1-0.90)^{x_{23}}\right)\left(1-(1-0.98)^{x_{31}}(1-0.92)^{x_{32}}\right) \\
\text { subject to } \tilde{4} x_{11} \oplus \widetilde{13} x_{12} \oplus \tilde{7} x_{13} \oplus \tilde{8} x_{21} \oplus \tilde{3} x_{22} \oplus \tilde{3} x_{23} \oplus \widetilde{11} x_{31} \oplus \tilde{5} x_{32} \preceq \widetilde{30} \\
\tilde{2} x_{11} \oplus \tilde{3} x_{12} \oplus \tilde{5} x_{13} \oplus \tilde{3} x_{21} \oplus \tilde{3} x_{22} \oplus \tilde{9} x_{23} \oplus \tilde{4} x_{31} \oplus \tilde{6} x_{32} \preceq \widetilde{17} \\
x_{11}+x_{12}+x_{13}+x_{21}+x_{22}+x_{23}+x_{31}+x_{32} \geq 1 \\
x_{11}, x_{12}, x_{13}, x_{21}, x_{22}, x_{23}, x_{31}, x_{32} \geq 0, \text { integers }
\end{gathered}
$$

Using the data in 3, FFNLIPP will be:

$$
\begin{gathered}
\text { Maximize } \tilde{R}_{\mathrm{s}} \approx\left(1-(1-\widetilde{0.99})^{\tilde{x}_{11}}(1-\widetilde{0.95})^{\tilde{x}_{12}}(1-\widetilde{0.92})^{\tilde{x}_{13}}\right) \otimes \\
\left(1-(1-\widetilde{0.98})^{\tilde{x}_{21}}(1-\widetilde{0.8})^{\tilde{x}_{22}}(1-\widetilde{0.90})^{\tilde{x}_{23}}\right) \otimes\left(1-(1-\widetilde{0.98})^{\tilde{x}_{31}}(1-\widetilde{0.92})^{\tilde{x}_{32}}\right) \\
\text { subject to } \tilde{4} \tilde{x}_{11} \oplus \widetilde{13} \tilde{x}_{12} \oplus \tilde{7} \tilde{x}_{13} \oplus \tilde{8} \tilde{x}_{21} \oplus \tilde{3} \tilde{x}_{22} \oplus \tilde{3} \tilde{x}_{23} \oplus \widetilde{11} \tilde{x}_{31} \oplus \tilde{5} \tilde{x}_{32} \preceq \widetilde{30} \\
\tilde{2} \tilde{x}_{11} \oplus \tilde{3} \tilde{x}_{12} \oplus \tilde{5} \tilde{x}_{13} \oplus \tilde{3} \tilde{x}_{21} \oplus \tilde{3} \tilde{x}_{22} \oplus \tilde{9} \tilde{x}_{23} \oplus \tilde{4} \tilde{x}_{31} \oplus \tilde{6} \tilde{x}_{32} \preceq \widetilde{17} \\
\tilde{x}_{11} \oplus \tilde{x}_{12} \oplus \tilde{x}_{13} \oplus \tilde{x}_{21} \oplus \tilde{x}_{22} \oplus \tilde{x}_{23} \oplus \tilde{x}_{31} \oplus \tilde{x}_{32} \succeq 1 \\
\tilde{x}_{11}, \tilde{x}_{12}, \tilde{x}_{13}, \tilde{x}_{21}, \tilde{x}_{22}, \tilde{x}_{23}, \tilde{x}_{31}, \tilde{x}_{32} \succeq 0, \text { integers }
\end{gathered}
$$

Using the data given in Tables 1, 2 and 3, crisp model as well as both the fuzzy models of the reliability optimization problem are formulated and solved according to the procedures discussed in the "Different defuzzification procedures". All the numerical problems are solved by optimization software LINGO 13 and the results are summarized in the Tables 4, 5, and 6 below.

\section{Conclusion}

In this manuscript, an $n$-stage series system with redundant units in parallel has been formulated as Non Linear Integer Programming Problem (NLIPP), in crisp and fuzzy environments. In fuzzy environment, imprecise parameters and decision variables are represented by triangular fuzzy numbers. Since the NLIPP in fuzzy environment cannot be 
Table 1 Input data for crisp model

\begin{tabular}{lllll}
\hline$j$ & Items & 1 & 2 & 3 \\
\hline \multirow{3}{*}{1} & $\tilde{c}$ & $(2,4,5)$ & $(6,8,9)$ & $(9,11,12)$ \\
& $\tilde{w}$ & $(1,2,4)$ & $(2,3,5)$ & $(3,4,6)$ \\
& $R$ & 0.99 & 0.98 & 0.98 \\
& $\tilde{c}$ & $(11,13,14)$ & $(1,2,4)$ & $(3,5,6)$ \\
2 & $\tilde{w}$ & $(2,3,5)$ & $(2,3,6)$ & $(5,6,8)$ \\
& $R$ & 0.95 & 0.8 & 0.92 \\
& $\tilde{c}$ & $(5,7,8)$ & $(1,3,4)$ & \\
3 & $\tilde{w}$ & $(4,5,7)$ & $(8,9,11)$ & \\
& $R$ & 0.92 & 0.90 & \\
$l_{i}$ & & 3 & 3 & 2 \\
\hline
\end{tabular}

Table 3 Input data for FFNLIPP as TFN

\begin{tabular}{lllll}
\hline$j$ & Items & 1 & 2 & 3 \\
\hline & $\tilde{c}$ & $(2,4,5)$ & $(6,8,9)$ & $(9,11,12)$ \\
1 & $\tilde{w}$ & $(1,2,4)$ & $(2,3,5)$ & $(3,4,6)$ \\
& $R$ & $(0.90,0.97,0.99)$ & $(0.95,0.98,0.99)$ & $(0.88,0.95,0.98)$ \\
& $\tilde{c}$ & $(11,13,14)$ & $(1,2,4)$ & $(3,5,6)$ \\
2 & $\tilde{w}$ & $(2,3,5)$ & $(2,3,6)$ & $(5,6,8)$ \\
& $R$ & $(0.85,0.95,0.99)$ & $(0.77,0.8,0.85)$ & $(0.85,0.92,0.97)$ \\
& $\tilde{c}$ & $(5,7,8)$ & $(1,3,4)$ & \\
3 & $\tilde{w}$ & $(4,5,7)$ & $(8,9,11)$ & \\
& $R$ & $(0.88,0.92,0.95)$ & $(0.85,0.90,0.97)$ & \\
$l_{i}$ & & 3 & 3 & 2 \\
\hline
\end{tabular}

Table 4 Optimal solution of crisp model

\begin{tabular}{lllllllll}
\hline$x_{11}^{*}$ & $x_{12}^{*}$ & $x_{13}^{*}$ & $x_{21}^{*}$ & $x_{22}^{*}$ & $x_{23}^{*}$ & $x_{31}^{*}$ & $x_{32}^{*}$ & $R_{s}^{*}$ \\
\hline 1 & 0 & 0 & 1 & 0 & 0 & 0 & 2 & 0.96399087 \\
\hline
\end{tabular}

Table 5 Optimal solutions of FNLIPP (Type I) by different defuzzification methods

\begin{tabular}{llllllllll}
\hline Methods & $x_{11}^{*}$ & $x_{12}^{*}$ & $x_{13}^{*}$ & $x_{21}^{*}$ & $x_{22}^{*}$ & $x_{23}^{*}$ & $x_{31}^{*}$ & $x_{32}^{*}$ & $R_{s}^{*}$ \\
\hline $\begin{array}{l}\text { Ranking } \\
\text { function }\end{array}$ & 1 & 0 & 0 & 1 & 0 & 0 & 1 & 1 & 0.9686477 \\
GMIV & 1 & 0 & 0 & 1 & 2 & 0 & 1 & 0 & 0.9694238 \\
$\alpha$-cut & 3 & 0 & 0 & 2 & 1 & 0 & 0 & 2 & 0.9935195 \\
\hline
\end{tabular}

Table 6 Optimal solutions of FFNLIPP (Type II) by different defuzzification methods

\begin{tabular}{llll}
\hline FOS & $\begin{array}{l}\text { Ranking } \\
\text { function }\end{array}$ & GMIV & $\alpha$-cut \\
\hline$\tilde{x}_{11}^{*}$ & $(5,7,1)$ & $(1,3,1)$ & $(1,0,1)$ \\
$\tilde{x}_{12}^{*}$ & $(0,0,0)$ & $(1,3,1)$ & $(0,0,2)$ \\
$\tilde{x}_{13}^{*}$ & $(0,0,1)$ & $(1,3,1)$ & $(1,1,2)$ \\
$\tilde{x}_{21}^{*}$ & $(3,4,2)$ & $(1,3,1)$ & $(1,1,2)$ \\
$\tilde{x}_{22}^{*}$ & $(2,2,1)$ & $(1,2,1)$ & $(1,1,1)$ \\
$\tilde{x}_{23}^{*}$ & $(0,0,1)$ & $(1,2,1)$ & $(0,1,2)$ \\
$\tilde{x}_{31}^{*}$ & $(4,7,1)$ & $(3,8,3)$ & $(0,1,4)$ \\
$\tilde{x}_{32}^{*}$ & $(0,1,3)$ & $(2,0,2)$ & $(1,1,4)$ \\
FOV & $(0.9999995$, & $(0.9995599$, & $(0.9155869$, \\
& 0.9999999, & 0.9999999, & 0.91816058, \\
& $0.9991818)$ & $0.9995599)$ & $0.99999903)$ \\
$\tilde{R}_{s}^{*}$ & 0.999795275 & 0.999853302 & 0.99999903 \\
\hline
\end{tabular}

FOS Fuzzy optimal solutiony, FOV Fuzzy optimal value

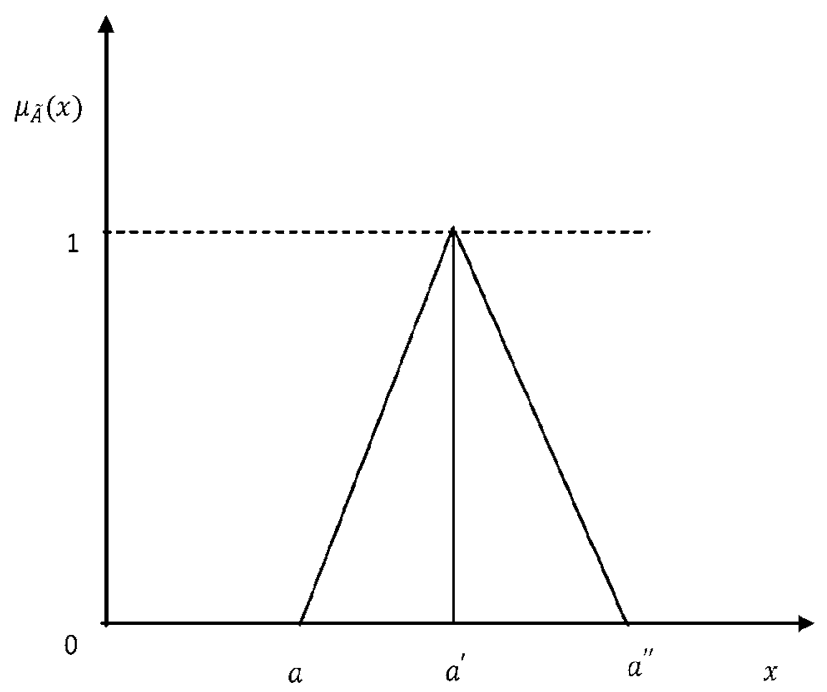

Fig. 1 Membership function of Triangular Fuzzy Number 
Fig. 2 Graphical comparison of optimal solutions of FNLIPP and FFNLIPP with NLIPP

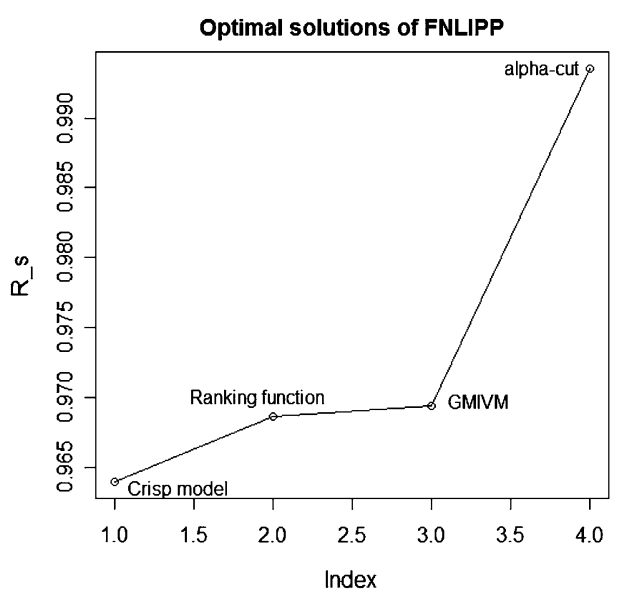

solved by any standard methods, therefore, the formulated problems has been defuzzified into crisp NLIPP using three different defuzzification methods viz. ranking function, graded mean integration value and $\alpha$-cut. An illustrative example has been solved to demonstrate the problem and solution procedure. From computational results, it is observed that the optimal system reliability obtained from $\alpha$-cut procedure is maximum in comparison to that of ranking function procedure, GMIV procedure and crisp model. Graphical representation of the results has been shown by the Figs. 1, 2 .

Open Access This article is distributed under the terms of the Creative Commons Attribution 4.0 International License (http://crea tivecommons.org/licenses/by/4.0/), which permits unrestricted use, distribution, and reproduction in any medium, provided you give appropriate credit to the original author(s) and the source, provide a link to the Creative Commons license, and indicate if changes were made.

\section{References}

Aggarwal S, Sharma U (2013) Fully fuzzy multi-choice multiobjective linear programming solution via deviation degree. Int $\mathrm{J}$ Pure Appl Sci Technol 19(1):49-64

Bansal A (2011) Trapezoidal fuzzy number (a, b, c, d): arithmetic behavior. Int J Phys Math Sci 2:39-44

Bector CR, Chandra S (2005) Fuzzy mathematical programming and fuzzy matrix games. Springer, Germany

Bortolan G, Degani R (1985) A review of some methods for ranking fuzzy numbers. Fuzzy Sets Syst 15:1-19

Bourezg A, Meglouli H (2015) Reliability assessment of power distribution systems using disjoint path-set algorithm. J Ind Eng Int 11(1):45-57

Chen S, Hsieh C (1999) Graded mean integration representation of generalized fuzzy numbers. J Chin Fuzzy Syst Assoc 5(2):1-7

Chern MS, Jan RH (1986) Reliability optimization problems with multiple constraints. IEEE Trans Reliab 35:431-436

Dolatshahi-Zand A, Khalili-Damghani K (2015) Design of scada water resource management control center by a bi-objective redundancy allocation problem and particle swarm optimization. Reliab Eng Syst Saf 133:11-21
Hsieh C, Chen S (1999) Similarity of generalized fuzzy numbers with graded mean integration representation. In: Proceedings of 8th International fuzzy System Association World Congress, vol 2, pp 551-555

Huang H (1996) Fuzzy multi-objective optimization decision-making of reliability of series system. Microelectron Reliab 37(3):447-449

Khalili-Damghani K, Abtahi A-R, Tavana M (2013) A new multiobjective particle swarm optimization method for solving reliability redundancy allocation problems. Reliab Eng Syst Saf 111:58-75

Khalili-Damghani K, Abtahi A-R, Tavana M (2014) A decision support system for solving multi-objective redundancy allocation problems. Qual Reliab Eng Int 30(8):1249-1262

Khalili-Damghani K, Amiri M (2012) Solving binary-state multiobjective reliability redundancy allocation series-parallel problem using efficient epsilon-constraint, multi-start partial bound enumeration algorithm, and dea. Reliab Eng Syst Saf 103:35-44

Kumar A, Kaur J (2011) A new method for solving fuzzy linear programs with trapezoidal fuzzy numbers. J Fuzzy Set Valued Anal 3:103-118

Kuo W, Prasad V, Tillman F, Hwang C (2001) Optimal reliability design fundamentals and applications. Cambridge University Press, Cambridge

LINGO User Guide (2013) Lindo systems inc., Chicago

Liou TS, Wang MJ (1992) Ranking fuzzy numbers with integral value. Fuzzy Sets Syst 50:247-255

Mahapatra GS, Roy TK (2006) Fuzzy multi-objective mathematical programming on reliability optimization model. Appl Math Comput 174(1):643-659

Mahapatra GS, Roy TK (2009) Reliability evaluation using triangular intuitionistic fuzzy numbers arithmetic operations. World Acad Sci Eng Technol 50:574-581

Mahapatra GS, Roy TK (2011) Optimal redundancy allocation in series-parallel system using generalized fuzzy number. Tamsui Oxf J Inf Math Sci 27(1):1-20

Misra K (1986) On optimal reliability design: a review. Syst Sci 12(4):5-30

Park K (1987) Fuzzy apportionment of system reliability. IEEE Trans Reliab R 36:129-132

Pramanik S, Roy TK (2008) Multiobjective transportation model with fuzzy parameters: priority based fuzzy goal programming approach. J Transp Syst Eng IT 8(3):40-48

Rao MS, Naikan V (2014) Reliability analysis of repairable systems using system dynamics modeling and simulation. J Ind Eng Int 10(3): $1-10$ 
Sasaki ROT, Shingai S (1983) A new technique to optimze system reliability. IEEE Trans Reliab 32:175-182

Sen N, Sahoo L, Bhunia AK (2014) An application of integer linear programming problem in tea industry of barak valley of Assam, India under crisp and fuzzy environments. J Inf Comput Sci 9(2):132-140

Sung CS, Cho YK (2000) Reliability optimization of a series system with multiple-choice and budget constraints. Eur J Oper Res 127:159-171
Taghizadeh H, Hafezi E (2012) The investigation of supply chain's reliability measure: a case study. J Ind Eng Int 8(1):1-10

Tillman I, Hwang C, Kuo W (1980) Optimization of system reliability. Marcel Dekker, New York

Yusuf I (2014) Comparative analysis of profit between three dissimilar repairable redundant systems using supporting external device for operation. J Ind Eng Int 10(4):199-207

Zadeh LA (1965) Fuzzy sets. Inf Control 8:338-353 